\title{
Metacognitive Awareness Inventory (MAI): Adaptação e Validação da Versão Portuguesa
}

\section{Metacognitive Awareness Inventory (MAI): Adaptation and Validation of Portuguese Version}

\author{
Rui Bártolo-Ribeiro ${ }^{1}$, Mário R. Simões ${ }^{2}$ e Leandro S. Almeida ${ }^{3}$
}

\section{Resumo}

O Metacognitive Awareness Inventory (MAI) de Schraw e Dennison (1994) avalia duas dimensões metacognitivas de natureza geral nos adolescentes e adultos: o conhecimento da cognição, que inclui três subprocessos, e a regulação da cognição, que compreende cinco subprocessos. Neste estudo apresenta-se o processo de tradução e de adaptação do MAI à população portuguesa. A consistência interna nas dimensões conhecimento e regulação da cognição foi 0,82 e 0,90, respetivamente. Os resultados da Análise Fatorial Confirmatória sugerem um fator de segunda ordem com base na correlação dos resultados nas duas dimensões. A subescala conhecimento declarativo apresentou melhor validade relativa ao critério comparativamente com as demais subescalas. O MAI constitui-se um instrumento adaptado à realidade portuguesa, tanto para a avaliação da metacognição no global, como nas suas dimensões principais, permitindo apoiar a investigação relativa ao rendimento em processos de aprendizagem e formação na população adulta e de adolescentes.

Palavras-chave: metacognição, avaliação da metacognição, Metacognitive Awareness Inventory (MAI), psicometria, validade de critério

\begin{abstract}
The Metacognitive Awareness Inventory (MAI) of Schraw and Dennison (1994) assesses two general metacognitive dimensions in adolescents and adults: knowledge of cognition, which includes three subprocesses, and regulation of cognition, which has five subprocesses. This study shows the process of translation and adaptation of MAI to the Portuguese population. Internal consistency in the dimensions knowledge and regulation of cognition was 0.82 and 0.90, respectively. The results of Confirmatory Factor Analysis suggest a factor of second order based on the correlation of two main dimensions. The declarative knowledge subscale showed better criterion validity compared to the other subscales. The MAI constitutes an adapted instrument to the Portuguese reality both for the assessment of a global metacognition score and in their two main dimensions, allowing research into the performance in learning and training processes in adults and adolescents.
\end{abstract}

Keywords: metacognition, assessment of metacognition, Metacognitive Awareness Inventory (MAI), psychometrics, criterion validity

\footnotetext{
${ }^{1}$ ISPA - Instituto Universitário. Rua Jardim do Tabaco, 34, 1149-041 Lisboa. 937190942. rbartolo@ispa.pt

${ }^{2}$ CINEICC. Laboratório de Avaliação Psicológica e Psicometria. Faculdade de Psicologia e Ciências da Educação da Universidade de Coimbra. Rua do Colégio Novo Apartado 6153, 3001-802 COIMBRA. simoesmr@fpce.uc.pt

${ }^{3}$ Centro de Investigação em Educação (CIEd), Instituto de Educação da Universidade do Minho. Universidade do Minho, Campus Gualtar, 4700-057 Braga. leandro@ie.uminho.pt
} 


\section{Introdução}

Vários autores têm evidenciado o papel determinante da inteligência geral na predição do desempenho no exercício e/ou na formação profissional (Carretta \& Ree, 1995; Fernie, Nikčević, Georgiou, \& Moneta, 2009; Hsu, 2010; Lord, 1952; Ree \& Earles, 1991). Contudo, os estudos de meta-análise atribuem reduzida variância explicada aos aspetos cognitivos identitários dessa capacidade intelectual geral (Bertua, Anderson, \& Salgado, 2005; Salgado, Anderson, Moscoso, Bertua, \& de Fruyt, 2003), o que aliás pode estar em sintonia com o modelo da inteligência fluida - inteligência cristalizada que sugere a diferenciação progressiva das habilidades cognitivas a partir da adolescência, com a consequente perda progressiva da importância da inteligência geral face às aptidões mais específicas (Almeida, Guisande, Primi, \& Lemos, 2008; Cattell, 1971; Facon, 2007).

Apesar da comprovada importância da inteligência geral para o êxito na aprendizagem, existem outras variáveis relevantes para esse sucesso. Gully e Chen (2010) identificam outros grupos de variáveis responsáveis pelos resultados na formação para além da aptidão mental geral e das aptidões e competências específicas, como sejam, as características sócio-demográficas descritas por evidências físicas e observáveis (e.g., sexo, idade), os traços de personalidade e outros associados ao autoconceito, bem como os valores e interesses. Para estes autores, o grupo de variáveis que inclui a aptidão mental geral e as aptidões e competências específicas contempla, também, habilidades específicas que geralmente não são avaliadas nos processos de seleção de pessoal, mas que surgem nas pesquisas enquanto variáveis relacionadas com o rendimento na formação, como é o caso, por exemplo, da metacognição (Martinez, 2006; Paris \& Winograd, 1990; Schraw, 2010; Schraw \& Dennison, 1994).

A introdução do termo 'metacognição' nos estudos do desenvolvimento e da aprendizagem é atribuído a John H. Flavell (1928- ) a partir do termo "metamemory", também por ele desenvolvido. Para Flavell (1979), metacognição refere-se ao conhecimento dos alunos da sua própria cognição. Numa abordagem mais alargada, a metacognição pode ser definida como a consciência das próprias cognições e o seu controlo (Cannon-Bowers, Rhodenizer, Salas, \& Bowers, 1998; Flavell, 1987). Neste sentido, a metacognição inclui o conhecimento que o sujeito possui das suas competências e a capacidade para desenvolver um plano para alcançar um objetivo, assim como de avaliar a sua eficácia em o alcançar. $\mathrm{O}$ conhecimento da cognição corresponde ao que os sujeitos sabem sobre si mesmos, sobre as estratégias e as condições em que as estratégias são mais úteis na aprendizagem. Por outro lado, a regulação da cognição traduz o modo como os alunos planeiam, implementam estratégias, monitorizam, corrigem os erros de compreensão e avaliam o seu desempenho. A título exemplificativo, a própria realização dos trabalhos diários por parte dos estudantes pode mudar a perceção relativamente à aprendizagem, constituindo-se como uma ferramenta de monitorização na regulação da cognição (Ferreira, Veiga-Simão, \& Silva, 2014), assim como a perceção que têm da sua própria eficácia se constitui um requisito fundamental para o sucesso e perseverança em desafios dificeis que lhe são colocados (Guerrero, Pérez, Contreras, \& Veja, 2013).

A metacognição tem sido estudada sobretudo na sua relação com a aprendizagem em amostras escolares e, nalguns casos, relativamente a áreas específicas da aprendizagem (e.g., matemática, Efklides \& Vlachopoulos, 2012). Contudo, justifica-se igualmente o estudo da metacognição no âmbito da formação profissional pela expectativa de que os formandos com maiores competências metacognitivas conseguem aprender mais eficazmente e melhor monitorizar o seu progresso, identificando as áreas em que têm de melhorar e ajustar adequadamente a sua aprendizagem (Gully \& Chen, 2010; Sternberg, 1998).

Diferentes métodos têm sido utilizados para avaliar a metacognição, nomeadamente, observações (e.g., Veenman \& Spaans, 2005), entrevistas (e.g., Artzt \& Armour-Thomas, 1992; Zimmerman \& Martinez-Pons, 1990), e questionários (e.g., Pintrich \& DeGroot 1990; Schraw \& Dennison 1994), entre outros. Todos 
estes métodos de avaliação da metacognição apresentam vantagens e inconvenientes associados ao contexto da investigação e da prática em que são utilizados. Contrariamente a outras formas de heteroavaliação dos processos metacognitivos com grande dispêndio de tempo e muito exigentes por parte dos examinadores, como é o caso do recurso a entrevistas (Artzt \& Armour-Thomas, 1992), os inventários de auto-avaliação são amplamente utilizados pela economia de recursos que representam.

Nas pesquisas efetuadas não foram identificados instrumentos construídos de raiz ou adaptados e validados para a população portuguesa que tenham sido originalmente desenvolvidos para avaliar a consciência metacognitiva na população adulta normal. Dinis e Gouveia (2011) estudaram as características psicométricas da versão portuguesa do Questionário de Metacognições (QMC-30; Metacognition Questionnaire de Wells \& Cartwright-Hatton, 2004), mas que mede as diferenças individuais numa seleção de crenças consideradas importantes no modelo metacognitivo de distúrbios psicológicos. Por outro lado, Gonçalves e Alves Martins (2013) para avaliarem a relação entre metacognição, autorregulação e utilização de estratégias cognitivas num contexto específico de ensino das ciências com uma amostra de alunos do $8^{\circ}$ ano, utilizaram o inventário da consciência metacognitiva Júnior. Este instrumento é uma adaptação do Jr. Metacognitive Awareness Inventory, criado por Sperling, Howard, Miller e Murphy (2002), a partir de um instrumento anteriormente criado por Schraw e Dennison (1994) para avaliação do desenvolvimento metacognitivo de adultos.

A não identificação de instrumentos de avaliação da metacognição construídos de raiz para a população adulta portuguesa, levou à pesquisa de instrumentos que pudessem vir a ser traduzidos e adaptados. O objetivo específico era identificar uma escala de medida da metacognição que pudesse ser utilizada em contextos de aprendizagem com adultos, como é o caso da formação profissional. A opção recaíu sobre o Metacognitive Awareness Inventory de Schraw e Dennison (1994).
O Metacognitive Awareness Inventory (MAI) foi originalmente desenvolvido por Schraw e Dennison (1994) para ser aplicado a adolescentes e adultos, com o objetivo de identificar o nível de consciência cognitiva de quem se encontra num processo de aprendizagem e satisfazendo três requisitos: (1) avaliar as componentes inerentes aos dois principais corpos teóricos relativos à metacognição (o conhecimento e a regulação da cognição; os 52 itens do MAI estão distribuídos por estes dois fatores metacognitivos); (2) sustentar a relação entre o conhecimento e a regulação da cognição, já que um dos pressupostos subjacentes às teorias da metacognição é estas componentes da cognição estarem mutuamente correlacionadas e serem compensatórias, prevendo-se igualmente que as soluções fatoriais obtidas pela rotação oblíqua dos fatores sejam mais favoráveis que as resultantes de rotações ortogonais (Baker, 1989; Brown, 1987; Flavell, 1987); e (3) relacionar as componentes da metacognição (conhecimento e regulação) com indicadores da capacidade de monitorização de um pré-teste, do desempenho numa prova, bem como com a capacidade de monitorizar a precisão do desempenho numa prova.

A versão inicial do MAI começou por ser constituída com 120 itens e incluiu um mínimo de 8 itens por cada uma das 8 componentes previamente definidas e agrupadas pelas dimensões conhecimento da cognição e regulação da cognição. Para Schraw e Dennison (1994), o conhecimento da cognição inclui três subprocessos: conhecimento declarativo (o conhecimento sobre si mesmo e sobre as estratégias), conhecimento processual (conhecimento sobre como usar estratégias) e conhecimento condicional (o conhecimento sobre quando e por que usar estratégias). Por sua vez, a regulação da cognição inclui cinco subprocessos que facilitam o controlo da aprendizagem: planeamento, estratégias de gestão da informação, monitorização da compreensão, estratégias de correção e avaliação (Artzt \& Armour-Thomas, 1992; Baker, 1989). Após eliminação dos itens com maior variabilidade nas respostas dadas por um grupo de estudantes universitários e dos itens que apareceram 
fortemente correlacionados entre si, foi obtida a versão final de 52 itens avaliando os oitos subprocessos metacognitivos já referidos.

As respostas na versão original eram dadas numa escala cujos itens são apresentados de forma bipolar, com $100 \mathrm{~mm}$ entre verdadeiro (extremo esquerdo) e falso (extremo direito). A opção de Schraw e Dennison (1994) por este formato de resposta resultou das vantagens relativamente às escalas do tipo Likert, nomeadamente uma maior proximidade às escalas contínuas e aumentando a variabilidade dos resultados. A Análise Fatorial Exploratória (AFE) foi realizada com e sem fixação do número de fatores, recorrendo ainda à rotação ortogonal e oblíqua dos fatores. As duas soluções proporcionaram resultados idênticos, ou seja, emergiram seis fatores com um valor próprio superior à unidade, sendo que os dois primeiros fatores agregaram a maior parte dos itens relativos ao conhecimento da cognição e os quatro fatores restantes incorporam a maioria dos itens relativos à regulação da cognição. As duas soluções da AFE não permitiram a identificação do modelo teórico inicial de 8 dimensões metacognitivas, ao mesmo tempo que os coeficientes alfa encontrados não foram satisfatórios para a maioria das escalas. Os autores decidiram então submeter os resultados a uma segunda AFE, definindo agora apenas dois fatores, como proposto por Brown (1987) e Jacobs e Paris (1987): um fator ligado ao conhecimento da cognição e outro fator relacionado com a sua regulação. Tanto a solução oblíqua como a ortogonal apontaram para o agrupamento dos itens pelos dois fatores, havendo apenas seis itens com uma saturação superior a 0,30 em ambos os fatores (itens 27, 34, 35, 40, 43 e 44) e dois itens não saturaram em nenhum dos fatores (itens 4 e 48). Os alfas de Cronbach obtidos para as duas dimensões foram já adequados (Schraw \& Dennison, 1994). A forte correlação entre os dois fatores significa que estas duas dimensões da metacognição operam em conjunto no processo de aprendizagem (Schraw \& Dennison, 1994). No que se refere à validade relativa ao critério, observa-se uma correlação positiva e estatisticamente significativa entre o rendimento dos participantes nas tarefas atribuídas e a dimensão conhecimento da cognição, mas a mesma tendência não se confirmou com a regulação. Schraw e Dennison (1994) efetuaram, também, uma comparação dos resultados do MAI com o rendimento na tarefa através da divisão da amostra em três grupos de desempenho (grupo 1, com desempenhos até ao percentil 60; grupo 2, com resultados entre percentis 61 e 75; e o grupo 3 , com resultados situados num percentil superior a 75). As ANOVAs realizadas permitiram identificar diferenças significativas entre os grupos 3 e 2, assim como 3 e 1; mas não foram encontradas diferenças entre os grupos 1 e 2 . Estas diferenças apenas foram verificadas para a dimensão conhecimento, sugerindo que não obstante as duas dimensões metacognitivas se encontrarem correlacionadas, partilhando entre 20 a $30 \%$ de variância comum, elas apresentam um comportamento diferenciado no que respeita à predição do rendimento na tarefa, deixando transparecer a ideia de que os aspetos que se relacionam com o rendimento não pertencem à variância comum.

Nesta pesquisa iremos relatar o processo de tradução e adaptação do MAI para a população portuguesa e apresentar os resultados relativos ao estudo das qualidades psicométricas da versão adaptada do Metacognitive Awareness Inventory (MAI) originalmente desenvolvido por Schraw e Dennison (1994). Foram testados dois modelos estruturais das diferentes escalas do inventário através da Análise Fatorial Confirmatória e estudada a validade relativa ao critério a partir das notas obtidas no último curso/ano referenciadas pelos participantes.

\section{Método}

\section{Participantes}

Considerando o objetivo do estudo na adaptação da escala para a população portuguesa, o questionário foi aplicado de modo direto a 900 participantes, por um processo de amostragem de conveniência tipo bola de neve, garantindo o empenho por parte dos respondentes, havendo apenas restrição ao nível do número de anos escolaridade, que deveria ser igual ou superior a 10. Foram rejeitados 16 questionários por não apresentarem respostas na segunda e/ou terceira página do questionário ou por terem sido identificados como outliers relativamente à 
proporção de respostas num dos pontos extremos da escala (superior a $75 \%$ ). Os 884 questionários considerados para estudo foram preenchidos por participantes com idades compreendidas entre os 15 e 50 anos (média $=21,87 ; D P=5,71$ ), dos quais $63,3 \%$ com idades comprrendidadas entre 18 e 22 anos, 9,7\% com idades inferiores a 18 anos, $17,9 \%$ entre os 23 e 27 anos e os restantes com idades superiores a 27 anos. A amostra é maioritariamente do sexo feminino $(72,2 \%)$. Os respondentes possuíam uma média de 13,2 anos de escolaridade $(D P=2,00)$, dos quais $46,8 \%$ possuíam entre o $10^{\circ}$ e o $12^{\circ}$ de escolaridade.

\section{Instrumento}

O Metacognitive Awareness Inventory (MAI) de Schraw e Dennison (1994) foi desenvolvido para ser aplicado a adolescentes e adultos, com o objetivo de identificar o nível de consciência cognitiva de quem se encontra num processo de aprendizagem. Composto por 52 itens respondidos numa escala bipolar, com $100 \mathrm{~mm}$ entre verdadeiro (extremo esquerdo) e falso (extremo direito). Schraw e Dennison (1994) obtiveram alfas de .88 para as duas dimensões (conhecimento da cognição e regulação da cognição) obtidas através da Análise Fatorial Exploratória, e uma correlação de .45 entre ambas.

\section{Processo de tradução}

Os itens foram traduzidos por quatro peritos portugueses fluentes em inglês, dos quais três psicólogos seniores e uma tradutora oficial não psicóloga. A retro-tradução para a língua original ficou a cargo de um profissional português residente no estrangeiro num país de língua oficial inglesa há mais de 10 anos. Os acordos entre tradutores foram conseguidos por consenso a partir da discussão num grupo de mestrandos em Psicologia e, posteriormente, validados pelos peritos. O processo de tradução incluiu também o inventário Memory and Reasoning Competence Inventory (Kleitman \& Stankov, 2007), que será utilizado no estudo da validade convergente.

\section{Descrição da versão portuguesa do MAI}

Foi organizado um questionário único formado a partir dos itens do Metacognitive
Awareness Inventory - MAI (Schraw \& Dennison, 1994) e do Memory and Reasoning Competence Inventory - MARCI (Kleitman \& Stankov, 2007): os primeiros 52 pertencem ao MAI e os últimos 16 ao MARCI. A decisão de juntar os dois questionários visou facilitar a recolha de respostas para o estudo da validade convergente. A totalidade dos itens está formulada na positiva e a resposta é dada numa escala de 5 pontos (1- Não se aplica rigorosamente nada a mim [0\%]; 2- Aplica-se pouco [25\%]; 3- Aplica-se em parte a mim [50\%]; 4- Aplica-se muito [75\%]; 5. Aplica-se completamente a mim [100\%]). A opção por uma escala de medida apoiada num referencial quantitativo (indicação de probabilidade de ocorrência) sustenta-se na expectativa de que a perceção da percentagem esperada de ocorrência do fenómeno no sujeito proporcione uma resposta com maior realismo do conceito qualitativo de cada um dos pontos da rating scale (e.g., Aplica-se muito) aproximandose da métrica utilizada na escala original por Schraw e Dennison (1994), que optaram pela técnica "escala visual analógica" e anteriormente descrita (ver DeVellis, 2003). Utilizou-se o software teleform $(\odot$ para a conceção gráfica dos inventários em papel e para poderem ser sujeitos a uma posterior leitura ótica.

Com os primeiros 52 itens são avaliadas as dimensões conhecimento e regulação da cognição. Dentro do conhecimento: conhecimento condicional (MAI_CK, 5 itens); conhecimento declarativo (MAI_DK, 8 itens); e o conhecimento processual (MAI_PK, 4 itens). Ao nível da regulação consideraram-se as variáveis: estratégias de correção (MAI_DS, 5 itens); avaliação (MAI_E, 6 itens); estratégias de gestão de informação (MAI_IMS, 10 itens), monitorização (MAI_M, 7 itens); e planeamento (MAI_P, 7 itens). Foi mantida a ordenação original dos itens na escala. Foram ainda considerados três scores compostos: a média global de consciência da metacognição (MAI_Tot) e que corresponde à média das respostas dadas nos 52 itens; a nota relativa à média para o conhecimento da cognição (KoC=Média das médias obtidas nas escalas MAI_CK; MAI_DK; e MAI_PK); e a nota relativa à média para a regulação da cognição 
(RoC=Média das médias obtidas nas escalas MAI_DS; MAI_E; MAI_IMS; MAI_M; e MAI_P). A pontuação global (MAI_Tot) reflete a perceção que os respondentes têm deles próprios relativamente a um contexto de aprendizagem não específico, assim como avalia a autoperceção das suas capacidades e estratégias no processo geral de aprendizagem, refletindo a visão mais abrangente que o sujeito tem do conhecimento e regulação da cognição.

As últimas 16 questões permitem a avaliação da autoperceção de duas componentes cognitivas específicas: a memória (MARCI_Mem) e o raciocínio (MARCI_Rac), e que poderíamos descrever como um "meta pensamento" sobre o próprio raciocínio e memória, que Kleitman e Stankov (2007) consideram como aspetos da metacognição.

No final do questionário foram colocadas questões demográficas (sexo, idade e número de anos de escolaridade frequentados), assim como uma pergunta para o estudo da validade relativa ao critério: "Média obtida no último ano/curso frequentado".

\section{Procedimento}

Foram utilizados questionários em papel que foram aplicados de modo presencial, individualmente e em grupo, por um conjunto de alunos do mestrado em psicologia, que receberam um briefing específico para a aplicação dos mesmos. Os objetivos do estudo foram apresentados aos participantes, e assegurada a confidencialidade dos resultados.

\section{Análise de dados}

Para validação da adaptação do MAI para a versão portuguesa, foi efetuada a análise da fiabilidade ao nível da consistência interna e o estudo de validade de constructo através da análise fatorial exploratória e confirmatória. Para garantia da consistência interna na fase de validação de instrumentos os alfas de Cronbach deverão ser superiores a 0,70 (Nunnally \& Bernstein, 1994). Para o estudo da validade de constructo através da AFE, foi analisada a qualidade das correlações entre as variáveis pelo teste de Bartlett e pela estatística de KaiserMeyer-Olkin (KMO). O teste Bartlett requer uma significância de $p<0,05$, enquanto o KMO deverá ter um valor de 0,7 ou superior para se assegurar uma recomendação média em se prosseguir com a AFE (Maroco, 2010b). A Análise Fatorial Exploratória foi efetuada como a versão 20.0 do SPSS e a Análise Fatorial Confirmatória (AFC) com recurso ao software AMOS (versão 20.0 do SPSS, IBM SPSS). Para estimar a validade convergente foi efetuada a correlação de Pearson com o inventário Memory and Reasoning Competence Inventory de Kleitman e Stankov (2007). No estudo de validade de critério foram efetuadas correlações momento-produto de Pearson e a Regressão Linear Múltipla.

Apesar de o modo de agrupamento dos itens pelos fatores estar teoricamente definido pelos autores do MAI, pelo que seria mais indicado tecnicamente efetuar diretamente a Análise Fatorial Confirmatória (Maroco, 2010a), optou-se por ensaiar, também, a AFE pelo método de Componentes Principais, considerando o carácter exploratório do estudo e o facto de ser a primeira aplicação da versão adaptada do MAI à população portuguesa. Face às controvérsias teóricas relativamente à fiabilidade do critério seguido para determinação do número de componentes a extraír da Análise de Componentes Principais (e.g., eigenvalue superior a um e observação gráfica do scree plot), foram também calculadas a análise paralela e média parcial mínima (minimum average partial - MAP) de Velicer ( $\mathrm{O}^{\prime}$ Connor, 2000). Na análise paralela é dada atenção ao número de componentes que contribuem com maior variância do que as componentes geradas a partir de dados aleatórios, enquanto a média parcial mínima toma em consideração a quantidade de variância retida na matriz de correlações após a extração do número crescente de componentes (O'Connor, 2000). Estes dois procedimentos não incluídos na versão 20.0 do Programa SPSS utilizado para a análise das componentes principais foram calculados a partir das propostas de O'Connor (2000) no ambiente de processamento matricial através do módulo syntax do SPSS.

\section{Resultados}

A Análise Fatorial Exploratória (AFE) foi efetuada através da Análise de Componentes 
Principais, sem restrição do número de fatores a considerar, com opção pela rotação oblíqua por se considerar que os itens das diferentes subescalas avaliam constructos relacionados à semelhança de outros estudos (Hsu, 2010; Larøi, Van der Linden, \& d'Acremont, 2009). Foi obtido um valor de Kaiser-Meyer-Olkin (KMO) para os 52 itens de 0,93 , o que associado ao facto de o teste de Bartlett ter uma significância de $p<0,001$, sustenta a realização da AFE (Maroco, 2010b).

Foram extraídas 12 componentes com um valor próprio superior à unidade e explicando $51,8 \%$ da variância total. A primeira componente extraída possui um poder explicativo de $22,1 \%$ da variância dos itens, sugerindo a existência de um fator dominante no questionário. A análise do scree plot dá uma indicação mais parcimoniosa dos fatores a serem considerados para interpretação (Figura 1), podendo-se considerar três soluções possíveis: um, três ou cinco fatores. As soluções sugeridas pela observação do scree plot não se adequam completamente ao modelo teórico, embora nos pareçam mais pertinentes que a obtida pelo critério de Kaiser. A opção pelo critério do scree plot relativamente ao de Kaiser está suportada pelo número de variáveis consideradas, que excede as 30 (Pestana \& Gageiro, 2003). Os resultados da análise paralela sugerem a extração de 5 componentes para interpretação, enquanto o método da média parcial mínima de Velicer (O’Connor, 2000) sugere a extração de 4 componentes. As soluções encontradas não diferem significativamente da opção tomada pelos autores com base na observação gráfica do scree plot.

No plano teórico, os autores Schraw e Dennison (1994) apresentam duas soluções possíveis: a de dois fatores (um com os itens das três subescalas do conhecimento da cognição, e outro com os itens das cinco subescalas relativas à regulação da cognição); e a de 8 fatores em que cada das subescalas se assume como um fator com identidade própria. Foram efetuados sucessivos ensaios com a extração forçada a diferente número de fatores a partir das soluções identificadas com a regra de Kaiser (valor próprio superior a 1,0), da observação gráfica do scree plot e das soluções propostas pelos métodos de análise paralelas e da média parcial mínima de Velicer (O’Connor, 2000),
Figura 1. Scree Plot da Análise de Componentes Principais ao MAI.

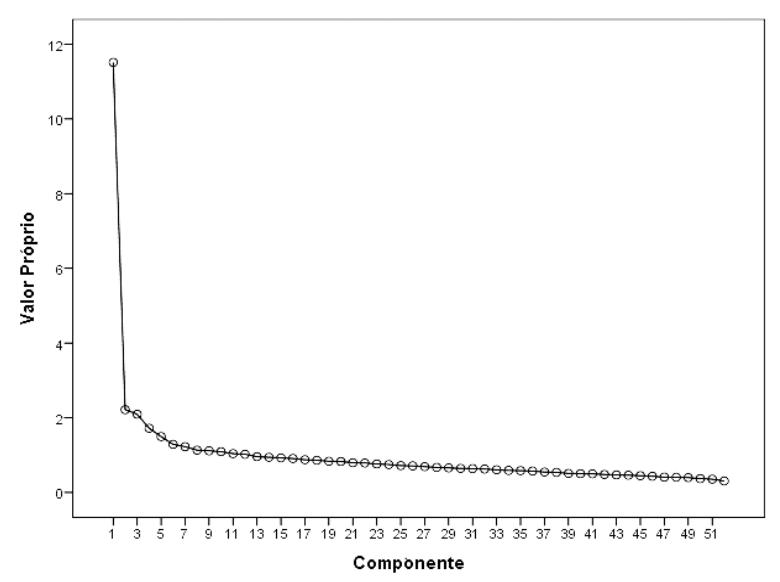

de modo a encontrar uma solução mais adequada de distribuição dos itens da escala na versão portuguesa pelas dimensões interpretáveis.

Nenhum dos ensaios com extração forçada a diferente número de fatores produziu resultados suficientemente satisfatórios e que pudessem caracterizar de modo estável a estrutura fatorial do MAI. A solução forçada a dois fatores mostra que 34 dos 52 itens apresentam um peso fatorial superior a 0,30 nas duas componentes, evidenciando a partilha de variância pelas duas dimensões. Na matriz de intercorrelações com os 52 itens encontramos todos os coeficientes de correlação positivos e um rácio de 1 coeficiente estatisticamente não significativo para 34 significativos $(\mathrm{p}<0,05)$, que sugere uma associação entre os itens. A percentagem de variância explicada pela primeira componente $(22,1 \%)$, comparativamente com a segunda (4,3\%), coloca-nos a hipótese de o MAI estar a avaliar, sobretudo, um fator metacognitivo geral. Stankov e Lee (2008) obtiveram idêntico resultado, pelo que utilizaram, no seu estudo, apenas os 10 itens que apresentaram os maiores pesos fatoriais nesse fator geral, com um alfa de 0,76 . Idêntico procedimento com a versão portuguesa assegura uma consistência interna de 0,80 envolvendo os 10 itens mais saturados na primeira componente extraída. Contudo, a extração forçada a 5 fatores permitiu identificar algumas tendências: a maioria dos itens relativos ao conhecimento da cognição satura no primeiro fator extraído; a maioria dos itens relativos à monitorização e à avaliação saturam num mesmo 
fator; e a totalidade dos itens relativos às estratégias de correção saturam num único fator.

Não havendo uma orientação clara, a partir da AFE para a reorganização estrutural dos 52 itens, efetuou-se a Análise Fatorial Confirmatória (AFC) com base no modelo proposto por Schraw e Dennison (1994) e evidenciado noutros estudos (e.g., Stankov \& Lee, 2008), testando dois modelos estruturais: (1) dois fatores latentes correlacionados (conhecimento e regulação da cognição); e (2) um fator latente de segunda ordem (consciência metacognitiva).

Os valores dos indicadores de ajustamento estão no nível mínimo de aceitabilidade (Maroco, 2010a) à exceção do Qui-quadrado e do CFI que estão acima de 2,0 e abaixo de 0,80, respetivamente (Quadro 1). Relativamente ao Quiquadrado, é um índice de ajustamento global e influenciado pelo tamanho da amostra tendendo a dar resultados significativos quando a amostra é superior a 200, como é o caso (Schumacker \& Lomax, 2010), contudo valores ponderados de Qui-quadrado/gl entre 2 e 5 são considerados sofríveis (Maroco, 2010a). Conforme consta do quadro 1, não se verificou uma diferença significativa nos índices de ajustamento entre os dois modelos.

Quadro 1. Indicadores de Ajustamento de dois Modelos do Metacognitive Awareness Inventory (MAI) pela Análise Fatorial Confirmatória

\begin{tabular}{ccc}
\hline $\begin{array}{c}\text { Indicadores de } \\
\text { ajustamento }\end{array}$ & $\begin{array}{c}\text { 2 Fatores } \\
\text { correlacionados }\end{array}$ & $\begin{array}{c}\text { Com fator de } \\
2^{\mathrm{a}} \text { ordem }\end{array}$ \\
\hline $\mathrm{X}^{2} / \mathrm{gl}$ & 3.401 & 3.403 \\
GFI & 0,815 & 0,815 \\
PGFI & 0,753 & 0,753 \\
CFI & 0,737 & 0,737 \\
PCFI & 0,708 & 0,708 \\
RMSEA & 0,052 & 0,052 \\
$\mathrm{P}(\mathrm{rmsea})<=0,05$ & 0,019 & 0,018 \\
\hline
\end{tabular}

Em termos de análise da consistência interna, o alfa de Cronbach da escala MAI com o total dos itens (52) foi de 0,93 , valor idêntico ao obtido por Kleitman e Stankov (2007). Foram identificados alfas menores em cada uma das duas dimensões e das oito subescalas. Na dimensão conhecimento foi obtido um alfa de 0,82 e os valores das subescalas variaram entre 0,53 e 0,67 ; para a dimensão regulação, o alfa alcançado foi de 0,90 e os alfas das 8 subescalas variaram entre $0,62 \mathrm{e}$ 0,73, conforme consta do Quadro 2.

Para o estudo da validade convergente do MAI foi utilizado o questionário MARCI, tendose efetuado dois procedimentos: a Análise de Componentes Principais (ACP) e as correlações momento-produto de Pearson com os totais obtidos nas várias subescalas (oito do MAI e duas do MARCI). Apesar de ambas as escalas serem autoavaliativas, possuem orientações de construção diferentes. Enquanto o MAI está centrado na avaliação de dois aspetos da metacognição: conhecimento sobre cognição e regulação da cognição; o MARCI está mais focado na avaliação da perceção que os respondentes possuem sobre a sua memória e raciocínio. Mesmo assim, estes dois questionários tendem a ser identificados como inventários metacognitivos (Stankov \& Lee, 2008).

As correlações entre as 10 subescalas são todas positivas e significativas a $p<0,01$ (Quadro 3 ), sugerindo que os dois inventários avaliam características psicológicas na mesma área (processos metacognitivos), confirmando a análise de Kleitman e Stankov (2007). Apesar da significância estatística das correlações encontradas, verifica-se que a maior percentagem de variância partilhada entre as duas escalas é com as subescalas do conhecimento da cognição e, em particular, com o conhecimento declarativo. Consequentemente, a proporção de variância partilhada é inferior quando consideramos as correlações entre as escalas do MARCI e as subescalas relativas à regulação da cognição, sobretudo em relação à subescala estratégias de correção.

Apesar de se poder pressupor a existência de correlação entre os fatores a partir dos resultados obtidos na matriz de intercorrelações entre as subescalas dos dois inventários, optou-se por efetuar uma ACP com rotação ortogonal por estarmos a lidar com itens de provas diferentes e com um número elevado de variáveis (52 itens do MAI mais 16 do MARCI). Os resultados obtidos através do KMO $(0,94)$ e do teste de esfericidade de Bartlett $(p<0,001)$ indicam que os valores são 
Quadro 2. Estatística Descritiva e Consistência Interna das escalas do Metacognitive Awareness Inventory (MAI)

\begin{tabular}{lcccc}
\hline & $\mathrm{k}$ & alfa & Média & D.-P. \\
Conhecimento da Cognição & & & & \\
Conhecimento Declarativo (DK) & 8 & 0,67 & 3,65 & 0,45 \\
Conhecimento Condicional (CK) & 5 & 0,57 & 3,67 & 0,51 \\
$\quad$ Conhecimento Processual (PK) & 4 & 0,53 & 3,55 & 0,53 \\
$\quad$ Subtotal Conhecimento da Cognição (KoC) & 17 & 0,82 & 3,62 & 0,43 \\
Regulação da Cognição & & & & \\
Planeamento (P) & 7 & 0,70 & 3,46 & 0,53 \\
Gestão da Informação (IMS) & 10 & 0,73 & 3,65 & 0,47 \\
Monitorização (M) & 7 & 0,66 & 3,45 & 0,51 \\
Estratégias de correção (DS) & 5 & 0,68 & 3,82 & 0,56 \\
Avaliação (E) & 6 & 0,62 & 3,45 & 0,54 \\
Subtotal Regulação da Cognição (RoC) & 35 & 0,90 & 3,57 & 0,43 \\
MAI - total & 52 & 0,93 & 3,59 & 0,40 \\
\hline
\end{tabular}

Nota. $\mathrm{N}=884 ; \mathrm{k}=\mathrm{n}^{\circ}$ de itens

Quadro 3. Intercorrelações entre as subescalas dos Inventários Metacognitive Awareness Inventory (MAI) e Memory and Reasoning Competence Inventory (MARCI)

\begin{tabular}{|c|c|c|c|c|c|c|c|c|c|c|c|}
\hline & & DK & CK & PK & $P$ & IMS & $M$ & DS & $E$ & Mem & Rac \\
\hline MAI-DK & Conhecimento Declarativo & - &, $651^{* *}$ &, $580^{* *}$ &, $564^{* *}$ &, $581^{* *}$ &, $537^{* *}$ &, $476^{* *}$ &, $471^{* *}$ &, $482^{* *}$ &, $487^{* *}$ \\
\hline MAI-PK & Conhecimento Processual & & & - &, $561^{* *}$ &, $553^{* *}$ &, $555^{* *}$ &, $451^{* *}$ &, $442^{* *}$ &, $274^{* *}$ & $379^{* *}$ \\
\hline MAI-P & Planeamento & & & & - &, $636^{* *}$ &, $631^{* *}$ &, $522^{* *}$ & $604^{* *}$ &, $312^{* *}$ &, $284^{* *}$ \\
\hline MAI-M & Monitorização & & & & & & - &, $507^{* *}$ & $690^{* *}$ & $205^{* *}$ &, $350^{* *}$ \\
\hline MAI-DS & Estratégias de correção & & & & & & & - &, $487^{* *}$ &, $112^{*}$ &, $195^{\star *}$ \\
\hline MAI-E & Avaliação & & & & & & & & - & ,208** &, $270^{* *}$ \\
\hline MARCI-Mem & Memória & & & & & & & & & - &, $447^{* *}$ \\
\hline
\end{tabular}

Figura 2. Scree Plot dos Valores Próprios da

Análise de Componentes Principais aos Inventários Metacognitive Awareness Inventory (MAI) e Memory and Reasoning Competence

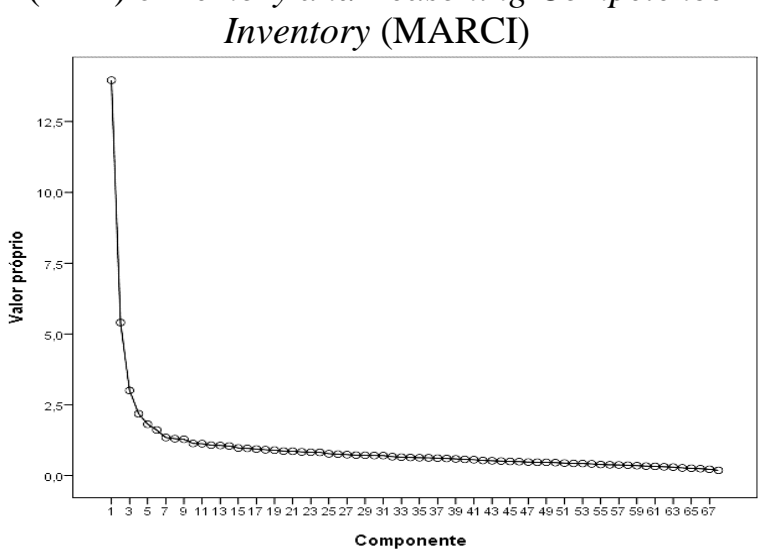

aceitáveis para prosseguir a análise e que provêm de uma população normal multivariada (Pestana \& Gageiro, 2003). A decisão do número de fatores a considerar para posterior interpretação foi tomada com base no scree plot (Pestana \& Gageiro, 2003). Complementarmente, a análise da matriz da variância explicada permitiu identificar que, a partir da sexta componente extraída, os incrementos de variância explicada são sempre inferiores a $0,06 \%$. Neste sentido, a ACP foi forçada à extração de seis fatores (Figura 2).

Os resultados revelaram a distribuição dos itens do MARCI nos dois primeiros fatores. O primeiro fator associado à memória e o segundo ao raciocínio. Nestes dois fatores dominados pelos 
Quadro 4. Pesos Fatoriais para a Análise Fatorial Exploratória com Rotação Varimax dos Itens dos Inventários Metacognitive Awareness Inventory (MAI) e Memory and Reasoning Competence Inventory (MARCI)

\begin{tabular}{|c|c|c|c|c|c|}
\hline & \multicolumn{5}{|c|}{ Componentes } \\
\hline & 1 & 2 & 3 & 4 & 5 \\
\hline Mem_68 &, 825 & & & & \\
\hline Mem_59 &, 786 & & & & \\
\hline Mem_55 & ,768 & & & & \\
\hline Mem_62 & ,766 & & & & \\
\hline Mem_57 &, 733 & & & & \\
\hline Mem_66 &, 723 & & & & \\
\hline Mem_53 &, 705 & & & & \\
\hline Mem_64 & ,698 & & & & \\
\hline DK_17 &, 655 & & & & \\
\hline IMS_13 & & ,597 & & & \\
\hline DK_12 & &, 574 & & & \\
\hline PK_27 & &, 522 & & & \\
\hline P_08 & &, 520 & & & \\
\hline P_04 & &, 496 & & & \\
\hline CK__26 & & ,488 & & & \\
\hline P_06 & & 474 & & & \\
\hline DK__10 & &, 474 & & & \\
\hline P_45 & & ,457 & & & \\
\hline PK__14 & &, 426 & ,346 & & \\
\hline IMS_30 & & ,399 & & & ,386 \\
\hline PK_33 & &, 393 & & & \\
\hline DK_05 & &, 377 & & & \\
\hline DK_20 & & ,375 & & & \\
\hline CK_35 & &, 361 &, 331 & & \\
\hline E_07 & & ,348 & & & \\
\hline IMS_09 & &, 344 & & & \\
\hline DK_16 & & ,339 & & & \\
\hline E_24 & & ,331 & & & \\
\hline \multicolumn{6}{|l|}{ M_02 } \\
\hline \multicolumn{6}{|l|}{ CK_18 } \\
\hline E_50 & & &, 723 & & \\
\hline M_49 & & & 669 & & \\
\hline E_38 & & & 632 & & \\
\hline E_19 & & &, 540 & & \\
\hline E_36 & & &, 505 & & \\
\hline P_22 & & &, 484 & & \\
\hline M_28 & & & ,446 & & \\
\hline M_21 & & & 417 & & \\
\hline IMS _41 & & & ,399 & & \\
\hline M_- 11 & & & ,376 & & \\
\hline M_01 & & &, 373 & & \\
\hline M_34 & & &, 343 & & \\
\hline P_23 & & ,331 &, 341 & & \\
\hline \multicolumn{6}{|l|}{ IMS_48 } \\
\hline Rac_61 & & & &, 775 & \\
\hline Rac_65 & & & & ,757 & \\
\hline Rac_58 & & & & ,698 & \\
\hline Rac_67 & & & & ,693 & \\
\hline Rac_63 & & & & ,675 & \\
\hline Rac_56 & & & &, 662 & \\
\hline Rac_54 & & & & 644 & \\
\hline Rac_60 & & & & ,628 & \\
\hline DK_46 & & & & & ,642 \\
\hline DS_52 & & & & &, 622 \\
\hline IMS_39 & & & & & 607 \\
\hline DS_51 & & & & &, 531 \\
\hline DS_25 & & & & &, 512 \\
\hline DS_44 & & &, 336 & &, 502 \\
\hline IMS_43 & & & & & ,465 \\
\hline CK_15 & & & & &, 451 \\
\hline DS_40 & & &, 374 & &, 448 \\
\hline IMS_37 & & & & & 441 \\
\hline IMS_31 & & & & & ,388 \\
\hline DK_32 & & & & & ,348 \\
\hline CK_29 & & & & & ,332 \\
\hline \multicolumn{6}{|l|}{ P_42 } \\
\hline IMS_47 & & & & & \\
\hline PK_03 & & & & & \\
\hline
\end{tabular}

Nota. Pesos fatoriais < 0,33 foram omitidos. Itens do Metacognitive Awareness Inventory (MAI): CK = Conhecimento Condicional; DK =

Conhecimento Declarativo; PK = Conhecimento Processual; DS = Estratégias de Correção; E = Avaliação; IMS = Estratégias de Gestão de Informação; $\mathrm{M}=$ Monitorização; P = Planeamento. Itens do Memory and Reasoning Competence Inventory (MARCI): MEM = Memória; RAC = Raciocínio. 
itens do MARCI figuram dois itens do MAI com pesos fatoriais superiores a 0,30: um item da subescala conhecimento declarativo da cognição no fator memória (peso fatorial $=0,67$ ) e um item relativo à subescala conhecimento condicional da cognição no fator raciocínio (peso fatorial $=0,31$ ). Embora as contribuições dos itens do MAI para explicação dos fatores memória e raciocínio sejam inferiores a qualquer um dos itens das dimensões respetivas do MARCI, os dados evidenciam esta proximidade entre o conhecimento declarativo e a memória e entre o conhecimento condicional e o raciocínio. O terceiro e sexto fatores são uma miscelânea de itens de diversas subescalas do MAI, não permitindo uma interpretação clara. Acresce o facto de alguns dos itens deste fator saturarem, também, noutros fatores com peso superior a 0,30. O quarto fator está ligado à regulação da cognição, englobando a totalidade dos itens da subescala estratégias de correção e 6 dos 10 itens da subsescala de estratégias de gestão de informação. O quinto fator também é possível ser interpretado, pois agrega quase exclusivamente os itens oriundos das subescalas monitorização e avaliação.

$\mathrm{O}$ facto de terem sido extraídos dois fatores não claramente interpretáveis, levou à realização de uma nova ACP com uma extração forçada a cinco fatores, numa tentativa de forçar a junção num só fator, os itens não saturados nos restantes fatores interpretáveis. $\mathrm{O}$ resultado permitiu uma interpretação mais consistente (Quadro 4). O primeiro fator mantém a configuração anterior, sendo facilmente interpretado como um fator de autoperceção da memória. $\mathrm{O}$ segundo fator agrega vários itens do MAI das várias subescalas pelo que pode ser designado por um fator metacognitivo não específico. $\mathrm{O}$ fator três junta a maioria dos itens de monitorização e avaliação e inclui dois itens relativos ao planeamento (22. Antes de começar, interrogo-me sobre a matéria; e 23. Penso nas várias formas de resolver um problema e escolho a melhor opção) e um item da subescala estratégias de gestão de informação (41. Uso a estrutura organizativa do texto para me ajudar a aprender). $\mathrm{O}$ quarto fator corresponde à dimensão raciocínio do MARCI. Tanto no fator 4 como no 1 , os itens que explicam este fator não apresentam saturação superior a
0,30 em qualquer outra dimensão, dando pureza de significado a estes fatores. O fator cinco está ligado à regulação da cognição, englobando essencialmente itens das subescalas estratégias de correção e estratégias de gestão de informação. Nesta última componente estão incluídos itens originários de outras subescalas do MAI como sejam: conhecimento declarativo da cognição (46. Aprendo mais quando o tema me interessa; e 32. Sei avaliar bem a minha compreensão das coisas); conhecimento condicional da cognição (15. Aprendo melhor, quando já sei alguma coisa sobre o assunto; e 29. Uso os meus pontos fortes intelectuais para compensar os meus pontos fracos); e planeamento (42. Eu leio cuidadosamente as instruções antes de começar uma tarefa).

No fator relativo à autoperceção da memória dominado pelos oito itens do MARCI satura um item do MAI com peso fatorial superior a 0,30, relativo ao conhecimento declarativo da cognição. Embora a contribuição deste item do MAI para explicação do fator autoperceção da memória seja inferior a qualquer um dos itens respetivos do MARCI, apresenta um peso fatorial significativo $(0,67)$. A análise do conteúdo do item em causa confirma esta proximidade entre o conhecimento declarativo e a memória (item 17 do MAI: sou bom a recordar informações).

Para estudo da validade de critério considerou-se a resposta dos participantes à questão "Média obtida no último ano/curso frequentado", colocada no final do questionário juntamente com outras questões demográficas. $\mathrm{O}$ valor da média foi expresso numa escala de 10 a 20 valores correspondendo à quantificação da variável critério. Os coeficientes de correlação encontrados são todos positivos e estatisticamente significativos, apesar da magnitude dos coeficientes ser relativamente baixa (Quadro 5). Os resultados corroboram os encontrados pelos autores do MAI, em que obtiveram uma correlação de $0,26(\mathrm{p}<0,05)$ entre a performance em quatro testes de compreensão de leitura e o conhecimento da cognição, mas somente 0,06 (n.s.) com a regulação da cognição (Schraw \& Dennison, 1994). Existem, contudo, diferenças metodológicas entre os estudos de validade de critério dos resultados no inventário. Enquanto 
Schraw e Dennison (1994) conceberam um estudo de validade concorrente e utilizaram como critério uma tarefa específica de compreensão de leitura, no presente estudo foi feita uma abordagem "pósditiva" (ver Kline, 2005), com a utilização de uma variável critério não diretamente associada a uma tarefa específica.

Quadro 5. Correlações de Pearson com o Critério "média no último ano/curso"

\begin{tabular}{lc}
\hline \multicolumn{1}{c}{ Dimensões } & $r$ \\
\hline Conhecimento Declarativo (DK) &, $19 * * *$ \\
Conhecimento Condicional (CK) &, $12 * * *$ \\
Conhecimento Processual (PK) &, $13 * * *$ \\
Planeamento (P) &, $11 * *$ \\
Gestão da Informação (IMS) &, $12^{* *}$ \\
Monitorização (M) &, $15^{* * *}$ \\
Estratégias de correção (DS) &, $09 * *$ \\
Avaliação (E) &, $10^{* *}$ \\
Conhecimento da Cognição &, $18^{* * *}$ \\
(DK+CK+PK) &, $14 * * *$ \\
Regulação da Cognição &, $16 * * *$ \\
(P+IMS+M+DS+E) & \\
MAI_total & Nota. N=854; *** p<0,001; ** p<0,01; ${ }^{*}<0,05$
\end{tabular}

Considerando que todos os coeficientes de validade relativa ao critéro são positivos e estatisticamente significativos efetuou-se uma regressão linear múltipla, pelo método stepwise, para determinar o melhor conjunto de variáveis metacognitivas explicativas do rendimento na última experiência de aprendizagem ocorrida e a respetiva percentagem de variância explicada. Os resultados salientam o conhecimento declarativo como a variável metacognitiva primeiramente responsável pela explicação da variância da nota obtida no último ano/curso com uma percentagem de variância explicada de $4 \%$. A regressão múltipla mostra-nos, assim, que o contributo das variáveis metacognitivas para o sucesso obtido num ano ou curso é muito reduzido.

Considerando a heterogeneidade da amostra utilizada para o processo de adaptação do MAI relativamente ao número de anos de escolaridade e à idade, repetiu-se o procedimento de regressão, mas filtrando a amostra aos sujeitos com idades inferiores a 20 anos e com 12 ou menos anos de escolaridade. O modelo manteve-se, mas agora com uma capacidade explicativa de $8 \%$. No quadro 6 encontram-se as duas regressões efetuadas: com a amostra global e com a amostra com restrições de idade e de anos de escolaridade.

Quadro 6. Análise da Regressão Linear Múltipla com Critério "média no último ano/curso" em duas amostras (total e restrita em idade e escolaridade)

\begin{tabular}{|c|c|c|c|c|c|}
\hline \multicolumn{6}{|c|}{ Amostra A. Amostra total $(N=854)$} \\
\hline & $R$ & $R^{2}$ & $R^{2} A j$. & Inc. $R^{2}$ & F Inc. \\
\hline $1^{\circ}$ Passo & & & & & \\
\hline $\begin{array}{c}\text { Conhecimento } \\
\text { Declarativo }\end{array}$ & 0,193 & 037 & ,036 & ,037 & $33,084 * * *$ \\
\hline
\end{tabular}

Amostra B. Participantes com dades inferiores a 20 anos e 12 ou menos anos de escolaridade $(N=256)$

\begin{tabular}{cccccc}
\hline & $R$ & $R^{2}$ & $R^{2} A j$. & Inc. $R^{2}$ & F Inc. \\
\cline { 2 - 6 } $1^{\circ}$ Passo \\
$\begin{array}{c}\text { Conhecimento } \\
\text { Declarativo }\end{array}$ & 0,281 &, 079 &, 076 &, 079 & $21,842^{* * * *}$ \\
\hline \multicolumn{5}{c}{ Nota. *** $\mathrm{p}<0,001$}
\end{tabular}

\section{Discussão}

Schraw e Dennison (1994), autores da escala, apresentam duas soluções possíveis de estruturação fatorial do inventário: a de dois fatores (conhecimento da cognição e regulação da cognição); e a de 8 fatores em que cada das subescalas se assume como um fator com identidade própria. A emergência de uma primeira componente muito forte (explicando 22,1\% da variância total e cinco vezes superior à segunda componente extraída), a partilha de variância por itens de diferentes constructos e a baixa fiabilidade nas subescalas teoricamente formuladas, sugerem que o MAI seja um questionário de avaliação global da metacognição. Este dado diverge da suposição teórica das suas 8 subescalas consideradas por Schraw e Dennison (1994). A nota global do MAI, muito suportada pela primeira componente extraída na ACP, pode assumir-se como um bom indicador dos processos metacognitivos, como foi, aliás, utilizado noutros estudos (e.g., Kleitman \& Stankov, 2007; Stankov \& Lee, 2008), situação que pode justificar a 
escolha de um número bastante menor de itens para a sua avaliação (apenas os itens mais saturados nesse primeiro fator).

Neste sentido, o MAI poderá ser entendido como um questionário que avalia os processos metacognitivos do sujeito em termos globais. A AFC corrobora estes resultados, conseguindo um ajustamento mínimo e idêntico tanto para a solução de um fator latente de segunda ordem (processos metacognitivos) como para a solução das duas dimensões relacionadas (conhecimento e regulação da cognição). Ambas estas soluções legitimam a possibilidade do inventário poder ser utilizado na investigação e na prática para a obtenção de uma pontuação dos processos metacognitivos diferenciados pelo conhecimento e regulação da cognição, como defendem Schraw e Dennison (1994), ou de uma pontuação geral dos processos metacognitivos. Gonçalves, Fidalgo e Alves Martins (2011), no estudo de adaptação do Inventário da Consciência Metacognitiva Júnior (ICM Jr.) a partir do Jr. Metacognitive Awareness Inventory (Jr. MAI), criado por Sperling, Howard, Miller e Murphy (2002), desenvolvido a partir do Metacognitive Awareness Inventory de Schraw e Dennison (1994), propõem a sua utilização para avaliar os dois componentes como um todo.

O conhecimento declarativo da cognição diferenciou-se das restantes subescalas ao apresentar a maior correlação com o critério. Contudo, o facto de não ter havido uma validação independente da resposta à questão que operacionalizava o critério ("Média obtida no último ano/curso frequentado"), pode constituir uma limitação deste estudo. Em estudos futuros, uma definição objetiva do critério, assim como, a uniformização do mesmo para todos os participantes poderá incrementar a capacidade explicativa das variáveis metacognitivas envolvidas. $\mathrm{Na}$ presente pesquisa, obteve-se um aumento de $4 \%$ da capacidade explicativa do critério pela subescala conhecimento declarativo da cognição, quando se filtrou amostra em termos de idade (inferior a 20 anos) e escolaridade (menor ou igual a 12 anos de escolaridade). Desta forma, elevou-se a probabilidade de os participantes se referirem ao mesmo critério. Por outro lado, a segmentação da amostra global no grupo com menor faixa etária pode estar relacionado com a eficiência metacognitiva. De acordo com Palmer, David e Fleming (2014), os indivíduos com uma boa eficiência metacognitiva tem uma consciência mais discriminativa que será manifestada por relatos objetivos e relacionados com o seu rendimento. Palmer e colaboradores (2014) encontraram um decréscimo não significativo da eficiência metacognitiva relativa à memória com o aumento de idade. Noutras variáveis de autoperceção próximas da metacognição, como o locus de controlo e a atribuição, verifica-se uma tendência para os sujeitos percecionarem as causas de modo mais externo quanto maior a escolaridade (e.g., Faria, 2000). Num estudo com 1529 alunos de diferentes anos de escolaridade, Faria (2000) verificou que os alunos dos anos de escolaridade inferiores consideram as causas como mais internas e mais controláveis do que os alunos de anos de escolaridade superiores. O facto de no nosso estudo se ter obtido maior correlação entre a consciência metacognitiva e o desempenho na aprendizagem num grupo com idade e habilitações literárias mais baixas que a amostra global pode ser um indicador de que a eficiência metacongitiva vai dimunuindo com o incremento daquelas duas variáveis.

O estudo da validade relativa ao critério das diferentes dimensões metacognitivas avaliadas pelo MAI relativamente ao sucesso no último ano/curso frequentado espelha a relação positiva e significativa entre os aspetos metacognitivos considerados no MAI e o sucesso na aprendizagem numa amostra de adolescentes e adultos. Os coeficientes de validade obtidos, significativos mas não muito elevados, podem ser corrigidos para atenuação da menor fiabilidade encontrada nos preditores considerados (Nunnally \& Bernstein, 1994). Apesar de os resultados obtidos serem mais favoráveis que no estudo de Schraw e Dennison (1994) relativamente ao papel da regulação da cognição na explicação do sucesso na aprendizagem, os estudos não são totalmente comparáveis por seguirem métodos diferentes relativamente à obtenção e operacionalização da variável critério. Contudo, o padrão da relação entre o conhecimento da cognição e a sua regulação relativamente à correlação com a performance na aprendizagem é 
idêntico: o conhecimento da cognição possui maior capacidade explicativa do sucesso na aprendizagem comparativamente à regulação da cognição. No entanto, em estudos de carácter experimental é atribuída mais importância aos aspetos da regulação da metacognição, nomeadamente através da monitorização, que no contexto da aprendizagem é avaliada através da confiança que têm em recordar uma questão que tenham aprendido (Hertzog, Sinclair, \& Dunlosky, 2010). A avaliação da capacidade metacognitiva em adultos tem sido avaliada por intermédio de questões relativas ao grau de confiança que os sujeitos têm nas respostas dadas em tarefas específicas (e.g., Palmer et al., 2014). Faltam ainda estudos que suportem a utilização de questionários metacognitivos no contexto da aprendizagem de adultos, nomeadamente na formação profissional, apreciando a capacidade explicativa das dimensões da metacognição neste contexto.

A fiabilidade da versão portuguesa do inventário global é elevada $(0,93)$ e idêntica à encontrada noutros estudos (Kleitman \& Stankov, 2007; Schraw \& Dennison, 1994). Contudo, os valores de consistência interna para algumas das subescalas tomadas isoladamente são inferiores a 0,70, o recomendável para este tipo de instrumentos (Kline, 2005; Pestana \& Gageiro, 2003). Marín e Rojas-Barahona (2010) no seu estudo de adaptação e validação do Trait Thinking Questionnaire (O’Neil \& Schacter, 1997) com escalas avaliadoras de duas das dimensões metacognitivas (planeamento e monitorização) e duas motivacionais apresentaram resultados superiores, mas numa amostra com idade e escolaridade inferiores. Por sua vez, a Análise de Componentes Principais demonstra a partilha de variância por itens que, teoricamente, estariam a medir constructos diferentes (e.g., monitorização e avaliação; estratégias de correção e estratégias de gestão de informação). Estes dados sugerem a necessidade de futuros estudos tomando a especificidade e comunalidade do conteúdo de tais itens, nomeadamente em termos dos processos metacognitivos envolvidos.

Por último, a forte associação entre as subescalas monitorização e a avaliação, assim como entre as subescalas estratégias de correção e estratégias de gestão de informação, deve ser considerada para futuras análises, nomeadamente na reformulação da designação destas dimensões. Os resultados obtidos revelam uma associação percecionada entre os itens da monitorização e da avaliação, assim como entre os itens relativos à utilização de estratégias de correção e estratégias de gestão de informação, justificando futuras investigações sobre o conteúdo dos itens específicos destes processos metacognitivos.

Num texto recente, Efklides e Vlachopoulos (2012) lembram que, 30 anos após a introdução do conceito de metacognição por Flavell (1979), este constructo ainda é difícil de definir e mais ainda de o avaliar. Reconhecendo o bem fundado daquela afirmação, o presente estudo disponibiliza um instrumento traduzido e adaptado à realidade portuguesa para avaliação de aspetos metacognitivos em amostras de adolescentes e adultos, contribuindo, assim, para a investigação relativa ao rendimento em processos de aprendizagem e formação.

\section{Referências}

Almeida, L. S., Guisande, M. A., Primi, R., \& Lemos, G. (2008). Contribuciones del factor general y de los específicos en la relación entre inteligencia y rendimiento escolar. European Journal of Education and Psychology, 1, 5-16.

Artzt, A. F., \& Armour-Thomas, E. (1992). Development of a cognitive-metacognitive framework for protocol analysis of mathematical problem solving in small groups. Cognition and Instruction, 9(2), 137175. doi: 10.1207/s1532690xci0902_3

Baker, L. (1989). Metacognition, comprehension monitoring, and the adult reader. Educational Psychology Review, 1(1), 3-38. doi: 10.1007/bf01326548

Bertua, C., Anderson, N., \& Salgado, J. F. (2005). The predictive validity of cognitive ability tests: A UK meta-analysis. Journal of Occupational and Organizational Psychology, 78(3), 387-409. doi: 10.1348/096317905x26994

Brown, A. (1987). Metacognition, executive control, self-regulation and other more mysterious mechanisms. In F. E. Weinert \& 
R. Kluwe (Eds.), Metacognition, motivation, and understanding (pp. 65-116). Hillsdale, N.J.: Lawrence Erlbaum.

Cannon-Bowers, J. A., Rhodenizer, L., Salas, E., \& Bowers, C. A. (1998). A framework for understanding pre-practice conditions and their impact on learning. Personnel Psychology, 51(2), 291-320. doi: 10.1111/j.1744-6570.1998.tb00727.x

Carretta, T. R., \& Ree, M. J. (1995). Air Force Officer Qualifying Test validity for predicting pilot training performance. Journal of Business and Psychology, 9(4), 379-388. doi: 10.1007/bf02230977

Cattell, R. B. (1971). Abilities, their structure, growth and action. Boston: Houghton Mifflin.

DeVellis, R. F. (2003). Scale development: Theory and applications (2nd ed.). Thousand Oaks, CA USA: Sage Publications.

Dinis, A., \& Gouveia, J. P. (2011). Estudo das características psicométricas da versão portuguesa do Questionário de Metacognições - versão reduzida e do Questionário de Metapreocupação. Psychologica, 54, 281-308.

Efklides, A., \& Vlachopoulos, S. P. (2012). Measurement of metacognitive knowledge of self, task, and strategies in mathematics. European Journal of Psychological Assessment, 28(3), 227-239. doi: 10.1027/1015-5759/a000145

Facon, B. (2007). Sur la différenciation des aptitudes cognitives de l' énfant. Revue Européenne de Psychologie Appliquée, 57, 213-223.

Faria, L. (2000). Aspectos desenvolvimentais das atribuições e dimensões causais: Estudos no contexto português. Revista Iberoamericana de Diagnóstico y Evaluación - e Avaliação Psicológica, 9(1), 23-39.

Fernie, B. A., Spada, M. M., Nikčević, A. V., Georgiou, G. A., \& Moneta, G. B. (2009). Metacognitive beliefs about procrastination: Development and concurrent validity of a self-report questionnaire. Journal of Cognitive Psychotherapy, 23(4), 283-293. doi: 10.1891/0889-8391.23.4.283

Ferreira, P., Veiga-Simão, A., \& Silva, A. (2014). Does training in how to regulate one's learning affect how students report self- regulated learning in diary tasks?. Metacognition and Learning, 10(2), 199-230, doi:10.1007/s11409-014-9121-3

Flavell, J. H. (1979). Metacognition and cognitive monitoring: A new area of cognitive developmental Inquiry. American Psychologist, 34(10), 906-911.

Flavell, J. H. (1987). Speculations about the nature and development of metacognition. In F. E. Weinert \& R. H. Kluwe (Eds.), Metacognition, motivation and understanding (pp. 21-29). Hillsdale, N.J.: Lawrence Erlbaum.

Gonçalves, J., \& Alves Martins, M. (2013). Avaliação da metacognição, autorregulação e utilização de estratégias cognitivas em atividades de investigação. In Actas do XII Congresso Internacional Galego-Português de Psicopedagogia. Braga: Universidade do Minho, (pp. 4153-4615).

Gonçalves, J., Fidalgo, Z., \& Alves Martins, M. (2011). Avaliação do desenvolvimento metacognitivo de estudantes entre o sexto e o nono ano de escolaridade. In Actas do XI Congresso Internacional Galego-Portugués de Psicopedagoxia. A Coruña: Universidade dá Coruña (pp. 2453-2462).

Guerrero, A., Pérez, J., Contreras, M., \& Vega, H. (2013). Composición factorial de una escala de autoeficacia en conductas académicas en universitarios de ingeniería. Revista Iberoamericana de Diagnóstico y Evaluación - e Avaliação Psicológica, 35(1), 77-93.

Gully, S., \& Chen, G. (2010). Individual differences, attribute-treatment interactions, and training outcomes. In S. J. Kozlowski, E. Salas (Eds.), Learning, training, and development in organizations (pp. 3-64). New York, NY: Routledge/Taylor \& Francis Group.

Hertzog, C., Sinclair, S. M., \& Dunlosky, J. (2010). Age differences in the monitoring of learning: Cross-sectional evidence of spared resolution across the adult life span. Developmental Psychology, 46(4), 939-948. doi:10.1037/a0019812

Hsu, L.-L. (2010). Metacognitive Inventory for nursing students in Taiwan: Instrument development and testing. Journal of Advanced 
Nursing, 66(11), 2573-2581. doi: 10.1111/j.1365-2648.2010.05427.x

Jacobs, J. E., \& Paris, S. G. (1987). Children's metacognition about reading: Issues in definition, measurement, and instruction. Educational Psychologist, 22(3/4), 255.

Kleitman, S., \& Stankov, L. (2007). Selfconfidence and metacognitive processes. Learning \& Individual Differences, 17(2), 161-173. doi: 10.1016/j.lindif.2007.03.004

Kline, T. J. B. (2005). Psychological testing: A practical approach to design and evaluation. Thousand Oaks, CA US: Sage Publications.

Larøi, F., Van der Linden, M., \& d'Acremont, M. (2009). Validity and reliability of a French version of the Metacognitions Questionnaire in a nonclinical population. Swiss Journal of Psychology/Schweizerische Zeitschrift für Psychologie/Revue Suisse de Psychologie, 68(3), 125-132. doi: 10.1024/14210185.68.3.125

Lord, F. M. (1952). The relation of the reliability of multiple-choice tests to the distribution of item difficulties. Psychometrika, 17, 181-194. doi: 10.1007/bf02288781

Marín, C., \& Rojas-Barahona, C. A. (2010). Adaptación y validación del Cuestionario de Rasgos de Pensamiento de O'Neil y colaboradores: Metacognición y motivación en la solución de problemas. Revista Iberoamericana de Diagnóstico y Evaluación - e Avaliação Psicológica, 30(2), 9-33.

Maroco, J. (2010a). Análise de Equações Estruturais: Fundamentos teóricos, software \& aplicações. Pêro Pinheiro: ReportNumber.

Maroco, J. (2010b). Análise Estatística com o PASW Statistics (ex-SPSS). Pêro Pinheiro: ReportNumber.

Martinez, M. E. (2006). What Is Metacognition? Phi Delta Kappan, 87(9), 696-699.

Nunnally, J. C., \& Bernstein, I. H. (1994). Psychometric theory. New York, NY: McGraw-Hill.

O'Connor, B. P. (2000). SPSS and SAS programs for determining the number of components using parallel analysis and Velicer's MAP test. Behavior Research Methods, Instrumentation, and Computers, 32, 396-402.
O’Neil, H.F., Jr, \& Schacter, J. (1997). Test Specifications for Problem - Solving Assessment (CSE Tech. Rep. $\mathrm{N}^{\circ}$ 463). Los Angeles: University of California, CRESST.

Palmer, E. C., David, A. S., \& Fleming, S. M. (2014). Effects of age on metacognitive efficiency. Consciousness and Cognition: An International Journal, 28, 151-160. doi:10.1016/j.concog.2014.06.007

Paris, S. G., \& Winograd, P. (1990). How metacognition can promote academic learning and instruction. In B. Jones, L. Idol (Eds.), Dimensions of thinking and cognitive instruction (pp. 15-51). Hillsdale, N.J.: Lawrence Erlbaum

Pestana, M. H., \& Gageiro, J. N. (2003). Análise de dados para ciências sociais: A complementaridade do SPSS ( $3^{\mathrm{a}}$ ed.). Lisboa: Edições Sílabo.

Pintrich, P. R., \& de Groot, E. V. (1990). Motivational and self-regulated learning components of classroom academic performance. Journal of Educational Psychology, 82(1), 33-40. doi: 10.1037/00220663.82.1.33

Ree, M. J., \& Earles, J. A. (1991). Predicting training success: Not much more than $\mathrm{g}$. Personnel Psychology, 44(2), 321-332.

Salgado, J. F., Anderson, N., Moscoso, S., Bertua, C., \& de Fruyt, F. (2003). International validity generalization of GMA and cognitive abilities: A European community metaanalysis. Personnel Psychology, 56(3), 573$605 . \quad$ doi: $\quad 10.1111 / \mathrm{j} .1744-$ 6570.2003.tb00751.x

Schraw, G. (2010). Measuring self-regulation in computer-based learning environments. Educational Psychologist, 45(4), 258-266. doi: 10.1080/00461520.2010.515936

Schraw, G., \& Dennison, R. S. (1994). Assessing metacognitive awareness. Contemporary Educational Psychology, 19, 460- 475.

Schumacker, R. E., \& Lomax, R. G. (2010). A beginner's guide to structural equation modeling ( $3^{\text {rd }}$ ed.). New York, NY US: Routledge/Taylor \& Francis Group.

Sperling, R. A., Howard, B. C., Miller, L. A., \& Murphy, C. (2002). Measures of children's knowledge and regulation of cognition. 
Contemporary Educational Psychology, 27(1), 51-79.

Stankov, L., \& Lee, J. (2008). Confidence and cognitive test performance. Journal of Educational Psychology, 100(4), 961-976.

Sternberg, R. J. (1998). Metacognition, abilities, and developing expertise: What makes an expert student? Instructional Science, 26, 127140.

Veenman, M. V. J., \& Spaans, M. A. (2005). Relation between intellectual and metacognitive skills: Age and task differences. Learning and Individual Differences, 15(2), 159-176. doi: 10.1016/j.lindif.2004.12.001

Wells, A., \& Cartwright-Hatton, S. (2004). A short form of the metacognitions questionnaire: Properties of the MCQ30. Behaviour Research and Therapy, 42(4), 385-396.

Zimmerman, B. J., \& Martinez-Pons, M. (1988). Construct validation of a strategy model of student self-regulated learning. Journal of Educational Psychology, 80(3), 284-290. doi: 10.1037/0022-0663.80.3.284

\section{Agradecimento}

Os autores agradecem a autorização dos autores das escalas originais para a sua utilização na realização deste estudo. 